\title{
Patent ductus arteriosus ligation versus medical therapy: A glowing recommendation for matching
}

\author{
Anusha Jegatheeswaran, $\mathrm{MD}, \mathrm{PhD},{ }^{\mathrm{a}}$ and Tara Karamlou, $\mathrm{MD}, \mathrm{MSc}^{\mathrm{b}}$ \\ From the a Division of Cardiovascular Surgery, Department of Surgery, Hospital for Sick Children, University of \\ Toronto, Toronto, Ontario, Canada; and ${ }^{\mathrm{b}}$ Division of Cardiovascular Surgery, Department of Surgery, Rady \\ Children's Hospital, University of California, San Diego, Calif. \\ Disclosures: Authors have nothing to disclose with regard to commercial support. \\ Received for publication June 1, 2018; revisions received June 1, 2018; accepted for publication June 4, 2018; \\ available ahead of print July 17, 2018 \\ Address for reprints: Tara Karamlou, MD, MSc, Mayo Clinic, Division of Cardiac Surgery, Rady Children's \\ Hospital, 3020 Children's Way, San Diego, CA 92123 (E-mail: tarakaramlou@ gmail.com). \\ J Thorac Cardiovasc Surg 2018;156:1945-6 \\ $0022-5223 / \$ 36.00$ \\ Copyright (c) 2018 by The American Association for Thoracic Surgery \\ https://doi.org/10.1016/j.jtcvs.2018.06.018
}

In this month's article by Mashally and colleagues, ${ }^{1}$ they attempt to answer the question: What outcomes are associated with oral acetaminophen therapy, in comparison with immediate surgical ligation (as defined by the authors) in neonates with persistent patent ductus arteriosus (PDA). The authors performed a retrospective, noncontemporaneous comparison of 43 patients who underwent immediate surgical ligation in an earlier era with 49 patients who were treated with acetaminophen, in whom surgery was restricted to those who failed medical therapy in the later era. The prevalence of crossover to surgical ligation in the later era occurred in the majority ( $53 \%$ ) of patients. Controversy exists regarding optimum mode of therapy, indications, and timing of surgical intervention, and the authors should be congratulated on investigating this important question in our field. ${ }^{2}$ The fallibility of medical therapy is that it may be ineffective, thus delaying the resolution of the hemodynamic compromise associated with a large PDA, resulting in a decline in the health status of the infant. ${ }^{2}$ Conversely, the surgical complications of PDA ligation are relatively rare, with immediate benefits.

The study has merit, insofar as it suggests a potential paradigm shift in care pathways for this challenging population that may reduce the need for surgical intervention. Unfortunately, there are several important limitations to the study that reduce its utility and impact.

First, the authors are comparing a medical therapy with a surgical therapy, which is fraught with selection bias. That the surgical therapy was applied only after failure of medical therapy further compromises confidence in the conclusions. Moreover, the patients in epoch 1 and epoch 2 differ in critical ways that likely affect the prevalence and risk of adverse outcomes in the latter group. These include the fact that patients in epoch 2 had a larger shunt fraction as evidenced by PDA diameter and prevalence of celiac artery diastolic flow reversal, coupled with a longer duration of

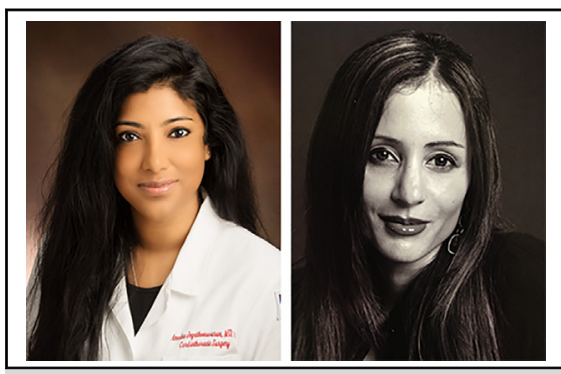

Anusha Jegatheeswaran, MD, PhD (left) and Tara Karamlou, MD, MSc (right)

Central Message

Comparison of medical with surgical therapy is hard because of selection bias. The study illustrates an important concept often minimized in the noninvasive era, but limitations undermine the conclusions.

See Article page 1937. exposure before treatment (12 vs 6 days, respectively). Intolerance to initial therapy with a cyclooxygenase- 1 inhibitor was higher in epoch 2 , and the treatment of lung disease of prematurity also evolved during the study period. In the absence of equipoise and a prospective randomized controlled trial, one avenue to mitigate confounding would be to use a propensity-matching algorithm (or even a propensity score) to account for the informative bias inherent in such nonrandomized comparisons.

The next issue that may magnify the impact of confounding by indication and suboptimal management of residual bias is that the authors have assigned morbidity (including chronic lung disease [CLD] and retinopathy of prematurity) to surgical ligation. Although previous studies have found that surgical ligation is associated with CLD, retinopathy of prematurity, and neurodevelopmental impairment in early childhood, ${ }^{3,4}$ we would contend that these reflect complications of prematurity in general and the natural history of this population. For instance, CLD in the setting of neonates with PDAs can be attributed to a prolonged period of pulmonary hypertension (exacerbated in patients in epoch 2). At the very least, these adverse outcomes could be due to the inherent inequality of the groups. Therefore, although we agree with the major finding of this study, "late acetaminophen therapy for infants with persistent PDA is associated with reduced 
surgical ligation but increased CLD," the study design undermines the validity of this conclusion. More traditional complications associated with surgical ligation were infrequent in the study population, but tended to increase in the later era, which also may speak to the increased acuity in this group. Reviewing the available literature, the most recent study in a large, contemporary cohort by Weisz and colleagues ${ }^{5}$ found that there was no difference in CLD or retinopathy of prematurity between medically or surgically managed preterm infants with PDA.

A final point to consider when interpreting the results of this study is that the dose of acetaminophen and the duration of treatment received by patients were variable. Therefore, it is unclear what the appropriate threshold would be for assigning a "failure" of medical therapy. Potential adoption or translation of the authors' findings would be increased by elucidation of a standardized protocol that clearly delineates criteria for failure of medical therapy.

Currently, every neonatal intensivist and pediatric cardiac surgeon is faced with understanding the tradeoffs between persisting with medical therapy in the hopes of PDA closure and proceeding to surgical ligation with almost immediate effect. The study by Mashally and colleagues ${ }^{1}$ is important because it focuses attention on the notion that avoidance/ delay of "invasive intervention," although a laudable end point, may cause more detrimental outcomes, especially in a physiologically fragile population. Therefore, it is unfortunate that the design and execution of this study compromise the strength of the conclusions. Although the authors, in our opinion, have crossed the finish line, perhaps they haven't played by all the rules.

\section{References}

1. Mashally S, Nield LE, McNamara PJ, Martins FF, El-Khuffash A, Jain A, et al. Late oral acetaminophen versus immediate surgical ligation in preterm infants with persistent large patent ductus arteriosus. J Thorac Cardiovasc Surg. 2018;156: 1937-44.

2. Lam JY, Lopushinsky SR, Ma IWY, Dicke F, Brindle ME. Treatment options for pediatric patent ductus arteriosus: systematic review and meta-analysis. Chest. 2015;148:784-93.

3. Knott EM, Zamora A, Cavett C, Wattsman TA. Decline in health status of extremely premature neonates with patent ductus arteriosus after second course of medical therapy. Eur J Pediatr Surg. 2013;23:270-2.

4. Weisz DE, More K, McNamara PJ, Shah PS. PDA ligation and health outcomes: a meta-analysis. Pediatrics. 2014;133:e1024-46.

5. Weisz DE, Mirea L, Rosenberg E, Jang M, Ly L, Church PT, et al. Association of patent ductus arteriosus ligation with death or neurodevelopmental impairment among extremely preterm infants. JAMA Pediatr. 2017;171:443-9. 